\title{
Employing Fractionally-Spaced Equalizers (FSE) for Magnetic Recording Channels
}

\author{
Piya Kovintavewat, M. Fatih Erden ${ }^{*}$, Erozan Kurtas*, and John R. Barry \\ Georgia Institute of Technology, Atlanta, GA 30332 \\ *Seagate Technology, 1251 Waterfront Place, Pittsburgh, PA 15222
}

We propose and investigate the performance of an FSE (oversampled) system in longitudinal and perpendicular recording channels. Results show that the proposed system outperforms the conventional (symbol rate) one whenever there is excess bandwidth, like in longitudinal recording.

System Model

The readback signal $r(t)$ in Fig. 1 can be written as

$$
r(t)=\sum_{k} b_{k} g\left(t-k T+\Delta t_{k}\right)+n(t),
$$

where $b_{k}=a_{k}-a_{k-1}$ takes on values $\{-2,0,2\}, a_{k} \in\{ \pm 1\}$ is a binary input sequence, and $n(t)$ denotes the AWGN with power $\sigma^{2}$. The transition response, $g(t)$, for longitudinal recording is $g(t)=1 /\left(1+\left(2 t / P W_{50}\right)^{2}\right)$, where $P W_{50}$ is the width of $g(t)$ at half its maximum, while that for perpendicular recording is $g(t)=\operatorname{erf}\left(\sqrt{\ln 16} t / P W_{50}\right)$ [1] where $\operatorname{erf}($.$) is an error$ function and $P W_{50}$ is the width of the derivative of $g(t)$ at half its maximum. The jitter noise $\Delta t_{k}$ is modeled with a truncated Gaussian probability distribution function with zero mean and variance $\sigma_{j}^{2}$. The readback signal is filtered by a low-pass filter whose cutoff frequency is at $N /(2 T)$, and then sampled assuming perfect synchronization (As shown in Fig. 1, $N=1$ corresponds to symbol rate). The received sequence, $z_{m}$, is equalized by a $T / N$-spaced equalizer $(N=1$ corresponds to a $T$-spaced equalizer (TSE)) such that $y_{k}$ resembles $d_{k}$. Finally, the VD performs Viterbi detection.

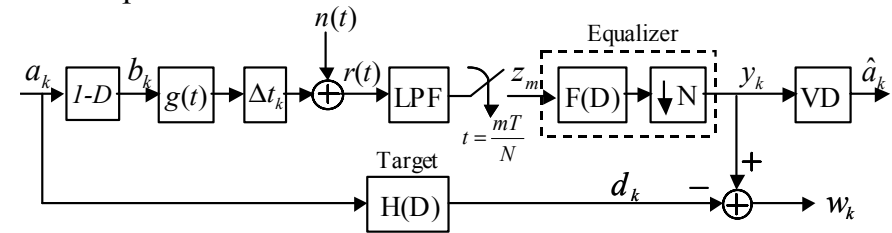

Fig. 1. Target and equalizer design

\section{Target and FSE Design}

Let $f_{k}$ and $h_{k}$ be coefficients of an $M$-tap equalizer and an $L$ tap target, respectively. The target and its FSE and/or TSE are designed to minimize $\mathrm{E}\left[w_{k}^{2}\right]=\mathrm{E}\left[\left\{\left(z_{N k} * f_{k}\right)-\left(a_{k} * h_{k}\right)\right\}^{2}\right]$ subject to the monic constraint [2]. This minimization process gives

$$
\begin{aligned}
& \lambda=1 /\left\{\mathbf{I}^{\mathrm{T}}\left(\mathbf{A}-\mathbf{P}^{\mathrm{T}} \mathbf{R}^{-1} \mathbf{P}\right)^{-1} \mathbf{I}\right\} \\
& \mathbf{H}=\lambda\left(\mathbf{A}-\mathbf{P}^{\mathrm{T}} \mathbf{R}^{-1} \mathbf{P}\right)^{-1} \mathbf{I} \\
& \mathbf{F}=\mathbf{R}^{-1} \mathbf{P} \mathbf{H}
\end{aligned}
$$

where $\lambda$ is Lagrange multiplier, $\mathbf{I}$ is an $L$-element column vector whose first element is one and the rest is zero, $\mathbf{A}, \mathbf{R}$ and $\mathbf{P}$ are $L$-by- $L, M$-by- $M$ and $M$-by- $L$ matrices with the $(i, j)$ th element $\mathbf{A}_{\mathrm{ij}}=\mathrm{E}\left[\sum_{k=0}^{S-1} a_{k-i} a_{k-j}\right], \mathbf{R}_{\mathrm{ij}}=\mathrm{E}\left[\sum_{k=0}^{S-1} z_{N k+K-i} z_{N k+K-j}\right]$ and $\mathbf{P}_{\mathrm{ij}}=\mathrm{E}\left[\sum_{k=0}^{S-1} z_{N k+K-i} a_{k-j}\right]$, respectively, $S$ is the length of input sequence $a_{k}, \mathbf{H}=\left[h_{0} h_{1} \ldots h_{L-1}\right]^{\mathrm{T}}$, and $\mathbf{F}=\left[f_{-K} \ldots f_{0} \ldots f_{K}\right]^{\mathrm{T}}$ where $[.]^{\mathrm{T}}$ is the transpose matrix operator. The resulting target is then known as a generalized partial response (GPR) target. As can be seen from the definitions of the matrices, equations (2), (3) and (4) reduce to their counterparts in [2] when $N=1$. Results

Fig. 2 (Left) compares the performance of the oversampled system $(N=2)$ and the symbol-rate system $(N=1)$ when $\sigma_{j} / T$ $=0 \%$ by plotting SNR required for BER $=10^{-4}$ as a function of the normalized recording density $\mathrm{ND}=P W_{50} / T$. We define $\mathrm{SNR}=10 \log _{10}\left(V^{2} / \sigma^{2}\right)$ in $\mathrm{dB}$ where $V^{2}$ is the signal energy (assumed to be 1). For each ND, the 5-tap GPR target and its corresponding 21-tap FSE and/or TSE were designed to minimize the SNR required to achieve the desired BER. A large gain is obtained in longitudinal recording if compared to perpendicular recording. This is because the longitudinal recording channel has large excess bandwidth than the perpendicular recording channel. We also compare two systems at $\sigma_{j} / T=9 \%$ using the GPR target obtained from the first experiment. Fig. 2 (Right) shows SNR required for BER $=10^{-3}$ as a function of ND. We again see a similar trend as in $\sigma_{j} / T=0 \%$ case. Note that the SNR required for $\mathrm{BER}=10^{-3}$ is very large in a perpendicular recording channel because media jitter noise at $\sigma_{i} / T=9 \%$ in this channel is very severe.
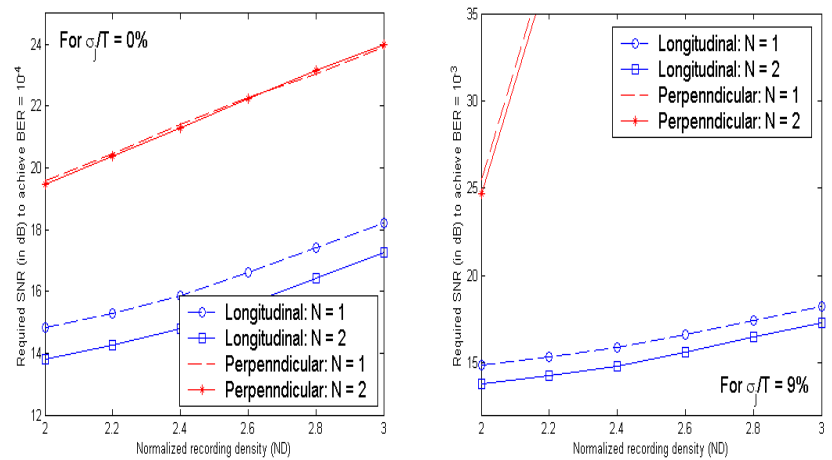

Fig. 2. Performance comparison of FSE against TSE References

[1] Piya Kovintavewat et al., "Generalized partial response targets for perpendicular recording with jitter noise," to appear in IEEE Trans. on Magnetics, September 2002.

[2] Jaekyun Moon and Weining Zeng, "Equalization for maximum-likelihood detectors," IEEE Trans. on Magnetics, vol. 31, no. 2, pp. 1083-1088, March 1995. 